\title{
ELECTROSTIMULATION OF THE HUUMAN EYE ADAPTED TO MONOCHROMATIC LIGHT, BY USING EXPONENTIALLY INCREASING CURRENT
}

\author{
MAKOTO SATO* \\ Physiological Laboratory, Iwate Medical College, Morioka, Iwate-ken, Japan
}

The accommodation process of the human retina to the electric current was first investigated by Motokawa and Iwama (3) by means of Hill's standard method using exponentially increasing currents as stimuli. Lately Sato, Suzuki and Akihama (5) studied again in detail the accommodation of the human retina adapted to various intensity levels of white light, by using exponentially increasing currents with varying time-constant $(\mathrm{RC})$ within a wide range from 0 to about $230 \mathrm{msec}$. They inferred that there would be within the retina two sorts of excitable tissues showing fast and slow accommodations, and that the one would be stimulated selectively by the increasing current of time-constant being shorter than $25 \mathrm{msec}$, and the other by that being longer than $90 \mathrm{msec}$.

Elaborating their experiment, I attempted to apply the experiment to the cases of the eye adapted to monochromatic lights of different wavelengths. The relationship between the electric threshold strength and the intensity of the adapting monochromatic light will be established precisely in this paper.

\section{METHODS}

A silver plate of $2 \times 3 \mathrm{~cm}$ in size was used as a stimulating electrode and it was inserted between the upper lip and gum. Another large silver plate was placed under the sole of the right foot as an indifferent electrode, being immersed into $1 \%$ saline. Fig. 1 shows the circuit diagram for measuring the electric threshold of the eye. Taking the electrical phosphene as an index of excitation, the electric thresholds $\left(V_{f}\right)$ for varying time-constants $(R C)$ were measured, where $V_{f}$ means the final voltage of the exponentially increasing current. The measuring procedure of the electric threshold $\left(V_{f}\right)$ was the same as stated in previous paper (5).

The adapting lights of different wavelengths were selected through Lange's metal interference filters, and their intensities were varied not only by using several well cali-

Received for publication October 12, 1957.

* 佐藤 誠

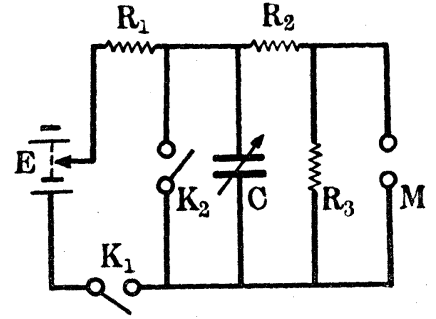

FIG. 1. Circuit diagram for producing exponentially increasing current.

$C$ : variable condenser $(0-$ $610 \mu F), E$ : battery (50 volts), $K_{1}$ : key, $K_{2}$ : contact key of pendulum, $R_{1}: 420$ ohms, $R_{2}: 3,000$ ohms, $M$ : subject. 
brated neutral tint filters of Matsuda SN-1 but also by a precise control of the supply voltage of the light source. For the details of the spectral transmittance of the interference filters I must refer the readers to Yaegashi's paper (8). The radiance $(N)$ of the surface of a light source for every adapting light was measured by a system of a thermopile (sensitivity: $5.1 \mathrm{~V}$ per watt) and a galvanometer (sensitivity: deflection of $1 \mathrm{~mm}$. at $1 \mathrm{~m}$. per $10^{-8} \mathrm{~V}$ ).

\section{RESUL,TS}

1) The relationship between the electric threshold $\left(V_{f}\right)$ and the radiance $(N)$

Variations in the electric threshold $\left(V_{f}\right)$ of the eye adapted to varying intensities of the radiance $(N)$, namely $V_{f}$ - $\log N$ curve, were investigated by using monochromatic rays with various wavelengths of 432, 463, 499, 550, 589 and $669 \mathrm{~m} \mu$ as the adapting lights. It was found that the form of $V_{f}$ - $\log N$ curve obtained was dependent not only on the wavelength of the adapting light but also on the time-constant $(R C)$ of the stimulating current. Accordingly, the form of the $V_{f}$ - $\log N$ curves for a given wavelength is dependent upon the time-constant of the stimulating current. Some examples for 550 and $669 \mathrm{~m} \mu$ in wavelength were shown in fig. 2 .

A

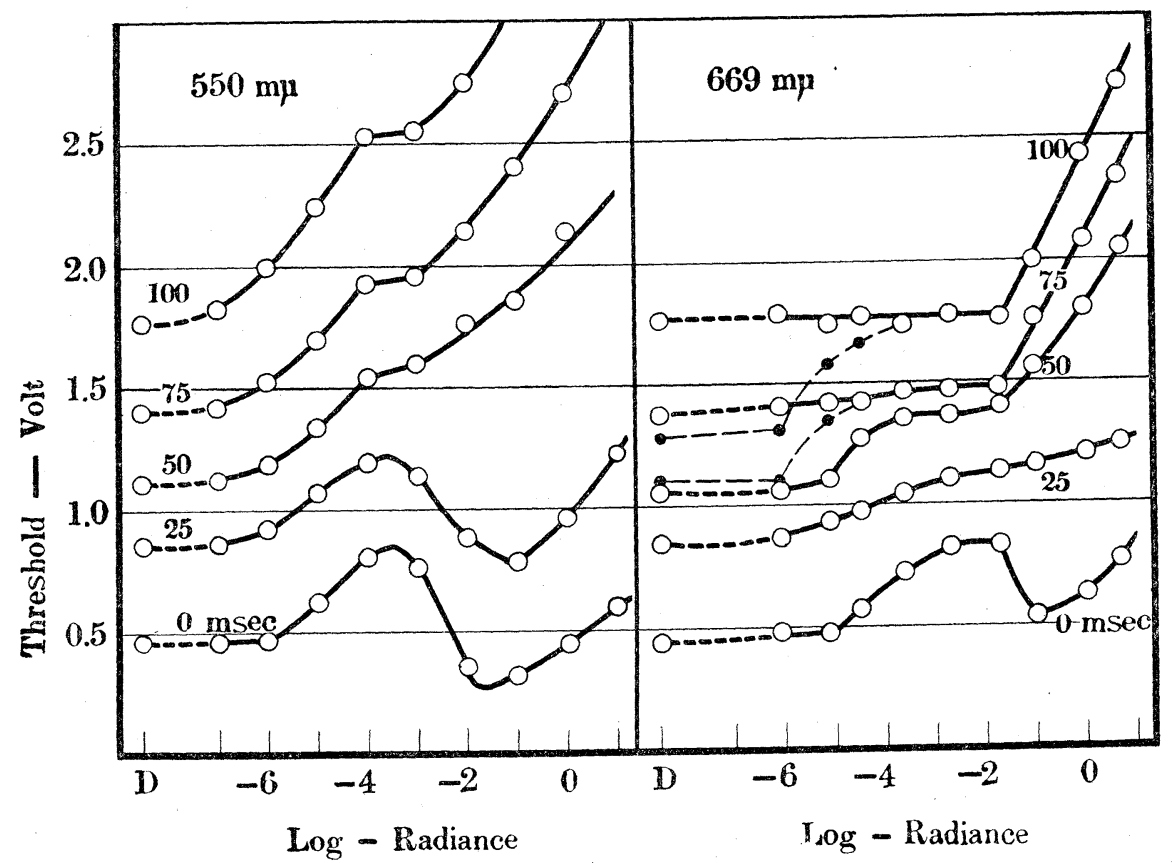

FIG. 2. Electric threshold strength-log-radiance relation. Numbers on the curves are time-constant of the current used. $D$ : dark. $(A)$ and $(B)$ are two families of curves obtained with the eye adapted to monochromatic lights of 550 and $669 \mathrm{~m} \mu$ in wavelength respectively. 
Fig. $2(A)$ shows that there are two different types of the $V_{f}-\log N$ curve depending on the different time-constant of the stimulating current, i.e., the one is a group of the curves for time-constant shorter than $25 \mathrm{msec}$, while the other is a group of those for time-constant longer than $75 \mathrm{msec}$. The value of the electric threshold for shorter time-constant increases at first gradually with the increase in intensity of the adapting light from dark to $10^{-3}$ (relative unit), till it arrives at a maximum, then it decreases rapidly to reach a minimum at $10^{-1}$ unit, and then it increases again progressively with further increase in radiance. On the other hand, the electric threshold for time-constant longer than $75 \mathrm{msec}$. increases with increase in radiance (hereafter this part on the curve is called "left slope"), till it arrives at a certain value of $V_{f}$ at which it is kept constant within a range between $10^{-4}$ and $10^{-3}$ unit (this part on the curve is called "horizontal section"). Again, a further increase in radiance causes the progressive rise in electric threshold $\left(V_{f}\right)$ (this part on the curve is called "right slope").

As for the subjective impression of the electrical phosphene, two different characteristics were perceived according to whether the time-constant was long or short. The electrical phosphene for time-constant shorter than $25 \mathrm{msec}$. appears in a twinkle as a faint flash of light, while that for time-constant longer than 50 msec. appears with a gradual rise in intensity and disappears with a gradual fall. All these results are well consistent with those of our previous experiment performed with eye adapted to white light. Therefore, I concluded that the characteristics of the $V_{f} \log N$ curve do not differ between the white light and the monochromatic ones, with an exception of $669 \mathrm{~m} \mu$.

Fig. $2(B)$ illustrates the $V_{f}$ - $\log N$ curves obtained for varying time-constants of the stimulating current under the adaptation to the monochromatic light of $669 \mathrm{~m} \mu$. As can be seen from comparison between fig. $2(A)$ and fig. $2(B)$, the curves for $669 \mathrm{~m} \mu$ are something different in shape from those for $550 \mathrm{~m} \mu$, especially in the cases of the stimulating currents with time-constant longer than $75 \mathrm{msec}$. For instance, the upper two curves presented in fig. 2 $(B)$ (curves connecting empty circles) run almost parallel to the horizontal axis within the range of radiance below $10^{-2}$ unit. In other words, these curves prove to have no "left slope", and that is the most remarkable difference from the curves for other shorter wavelengths. The value of the electric threshold $\left(V_{f}\right)$ begins to increase abruptly, marking a clear break on the curve, as the radiance is raised over about $10^{-2}$ unit.

As regards the electric threshold for relatively longer time-constant, it must be noted that another lower threshold can be determined, when Motokawa's comparing procedure (4) is employed for determination of electric threshold. Thereby, the other $V_{f}$ - $\log N$ curves (dotted ones connecting solid circles) were obtained, as shown in fig. $2(B)$. In this case, the impression of the electrical phosphene produced was that of a faint flash just identical with that observed usually in cases of time-constants shorter than $25 \mathrm{msec}$, notwithstanding that the time-constant used was actually longer than $75 \mathrm{msec}$. Such two values of the high and low thresholds had been reported already in the previous paper (5), but they were not so easily demonstrable as in the case of the adapted 
to monochromatic light of $669 \mathrm{~m} \mu$.

It is interesting to notice that two different values of the electric threshold correspond to two different impressions of the electrical phosphene, and that these facts can be confirmed most clearly in the case of illumination with such an extreme red ray as $669 \mathrm{~m} \mu$.

2) Dependence of $V_{f} \log N$ curve upon wavelength of the adapting light

As mentioned above, the form of the $V_{f} \log N$ curve is quite different according to whether the time-constant of the stimulating current is shorter than $25 \mathrm{msec}$. or longer than $75 \mathrm{msec}$. Therefore, both cases of time-constants at 0 and $100 \mathrm{msec}$. were specified as typical instances for systematic studies on the effect of wavelength of the adapting light on the $V_{f}$ - $\log N$ curve. Two series of the curves obtained by varying wavelengths in both cases of time-constants at 0 and $100 \mathrm{msec}$. were illustrated in fig. $3(A)$ and $(B)$ respectively; in the interest of a clear understanding of the graphical representation, each of these curves was illustrated by shifting vertically by a certain distance.

A

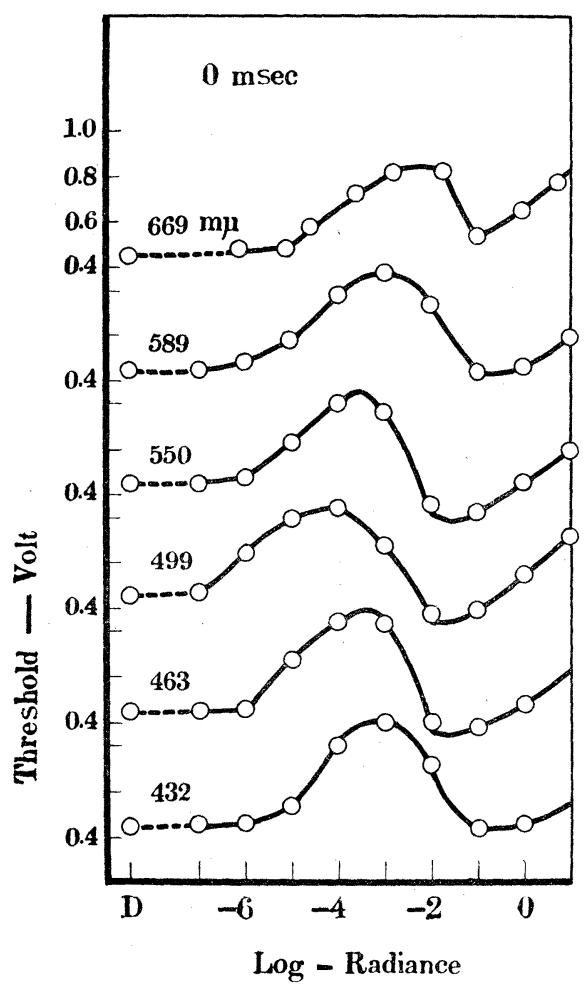

B

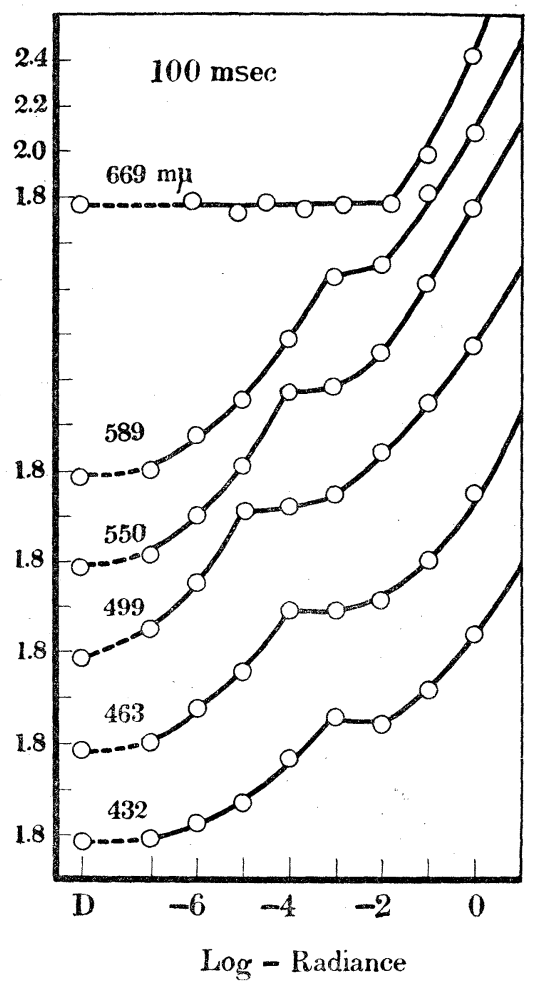

FIG. 3. Electric threshold strength-log-radiance relation. Numbers on the curves are wavelengths of the adapting light. $D$ : dark. Each curves is shifted vertically for graphical representation. $(A)$ and $(B)$ are two families of the curves obtained by stimulation with time-constant at 0 and $100 \mathrm{msec}$, respectively. 
Fig. $3(A)$ shows that the value of radiance (abscissa) corresponding to the crest (maximum) on the curve varies depending upon the wavelength, and that it takes a minimum at about $500 \mathrm{~m} \mu$ and increases toward both red and violet ends of the spectrum. Fig. $3(B)$ shows similarly to fig. $3(A)$ that the value of radiance corresponding to the beginning (or left edge) of the "horizontal section" varies depending upon the wavelength of the adapting light, and that it takes the lowest at about $500 \mathrm{~m} \mu$.

It is worth noticing that both of crest and the left edge of the "horizontal section" on the respective curves lie in the same point of abscissa (radiance) correspondingly to each other.

We can obtain a spectral sensitivity curve by taking the reciprocal of the radiance corresponding to the crest of the hump (or the left edge of the "horizontal section"), as the criterion. Fig. 4 shows that the data (filled circles) prove themselves to be consistent fairly well with the data of scotopic luminosity from Weaver (7). This finding indicates that luminosity at scotopic vision has a close relation to the formation of the crest on the $V_{f}$ - $\log N$ curve.

As for characteristic of the sensation produced by the adapting light, no hue could be elicited within any range of radiance corresponding to the ascending arm of the hump on the curve in fig. $3 .(A)$ or to the "left slope" of the curve in fig. 3 $(B)$. Hue appeared first at a radiance corresponding to the descending part toward the minimum of $V_{f}$ - $\log N$ curve in fig. $3(A)$, and the increase in saturation of colour takes place progressively with increase in radiance.

After all, it seems likely that

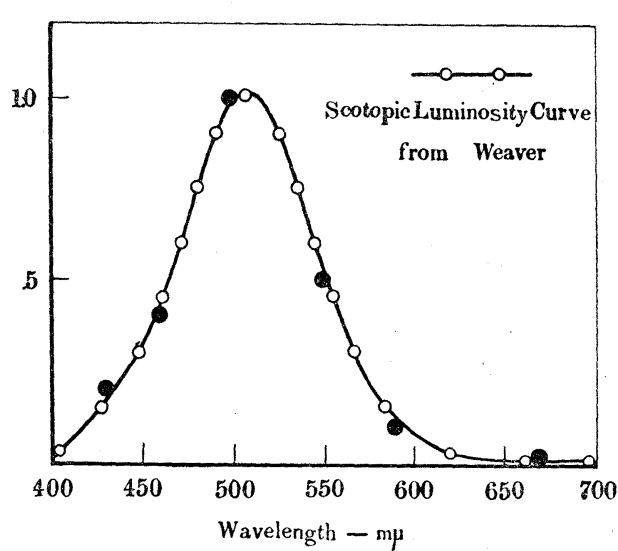

FIG. 4. Comparison of our data with Weaver's scotopic luminosity curve. Filled circles are plotted by taking reciprocal of the radiance corresponding to the crest on the curves in fig. $3(A)$. The curve connecting empty circles is the scotopic luminosity curve after Weaver. each of the "left slope" and the ascending arm of the hump in the respective curves relates exclusively to rod function, while each of the "horizontal section" and the descending arm of the hump in respective curves relates to concurrent participation of both cones and rods, and that the "right slope" and the ascending part transgressing the minimum may be concerned predominantly with cone function.

\section{DISCUSSION}

Sato, Suzuki and Akihama (5) studied the accommodation of the human retina to exponentially increasing currents at various intensity levels of white adapting light. They found that there were two different types of the electric 
threshold strength-log-illumination curves according to whether the time-constant of the stimulating current was shorter than $25 \mathrm{msec}$. or longer than $75 \mathrm{msec}$.

At almost the same time, Mita, Fujimaki and Yaegashi (2) obtained the same conclusion from the experiments performed with use of the sinusoidal alternating currents of very low frequency as the stimuli. From this conclusion they inferred that the difference between two types of the strength-log-illumination curves for both longer and shorter time-constants might be due primarily to different seats of excitation. They, furthermore, assumed that the structures stimulated by the current of longer time-constant might be the photoreceptor cells (primary neurons), while those stimulated by the current of shorter timeconstant might be the bipolar cells (secondary neurons).

As stated above, the present experiments corroborate the previous worker's findings in the main, namely, two familiar types of the strength-log-radiance curves were again demonstrated in all cases of adapting lights of different wavelengths excepting the extreme red end of the spectrum. However, it is worth noting the exceptional cases of red adapting light. All curves in fig. 3 $(A)$ resemble in shape each other, whereas the most upper curve in fig. $3(B)$ differs in shape from all the other curves in the same figure. Namely, in a special condition of experiment, where the time-constant of the stimulating current is $100 \mathrm{msec}$. and the adapting light consists of pure red rays of $669 \mathrm{~m} \mu$ in dominant wavelength, the electric threshold strength-log-radiance curve takes a singular form which can never be found in the case of white adapting light. In this case the red rays bring about the specific effect due to the preponderance of cone function, so that the single curve for the red rays exhibits the lack of the effect due to rod function. This fact indicates that the electric threshold in this experimental condition is determined independent of rod activities, if any, but dependent solely of cone activities.

In the case of $0 \mathrm{msec}$. in time-constant, however, the circumstance is quite different from the former case; strange to say the red rays have not such a specific effect, as has been found in the case of time-constant of $100 \mathrm{msec}$. As shown in fig. $3(A)$, the form of the curve for $669 \mathrm{~m} \mu$ is of duplex nature and in common with those of the other curves for different wavelengths. This fact indicates that the electric threshold for time-constant of $0 \mathrm{msec}$. depends always not only upon rod- but also on cone-activities, irrespective of the wave length of adapting light. After all, this difference in the effect of one and the same red rays upon the electric threshold in both cases of longer and shorter time-constants seems to be an evidence for supporting the view of different seats of excitation.

As the electric threshold means the intensity of the electric stimulus, which elicites the slightest light sensation just discriminative from brightness of the surrounding background (adapting light), it is regarded to have some relations to the differential threshold of brightness discrimination. If the electric threshold strength $(V)$ is assumed to be proportional to logarithm of the differential threshold $(\Delta I)$, that is, the least perceptible brightness increment to be added to the brightness $(I)$ of the background, then

$$
V=K \log (\Delta I)
$$


where $K$ is a proportionality constant. Denoting Weber's fraction by $W$,

$$
\log W=\log (\Delta I / I) \text {. }
$$

From (1) and (2),

$$
V=K \log (\Delta I)=K(\log W+\log I) .
$$

It is widely known that, as log $I$ increases, Weber's fraction ( $W$ ) decreases steadily and approrches a finite limiting value depending upon the size of the test-field. Some of the latest measurements of $W$ were made by Steinhardt (6) and Blackwell (1). Their data for larger teat-field exhibit a break in the curve in the section where $W$ decreases rapidly. Using their data of $\log W$ and $\log I$, we can calculate the values of $V$ by the formula (3). Thus, if plotted, we obtained a new $V \cdot \log I$ curve by calculation. When the calculated $V \cdot \log I$ curve was compared with $V_{f} \log N$ curves obtained in this experiment, the former curve agreed in shape fairly well with those curves obtained in the cases of longer time-constants longer than $75 \mathrm{msec}$., whereas it did not agree with those curves obtained in the cases of time-constants shorter than $25 \mathrm{msec}$. The disagreement in the latter cases seems to occur mainly in the descending arm of $V_{f}$ - $\log N$ curve; this part of the curve corresponds to the range of radiance where both rods and cones are active at the same time.

From the above mentioned facts, it may be concluded that the electric threshold determined by the exponentially increasing current of the time-constants longer than $75 \mathrm{msec}$. is equivalent to the differential threshold of brightness discrimination, while that determined by the current of time-constants shorter than $25 \mathrm{msec}$. is not. This conclusion also seems to give some credit to Mita et al.'s view of different seats of excitation.

\section{SUMMARY}

Taking, as a criterion, the electrical phosphene evoked by electrostimulation of the human eye with use of the exponentially increasing currents of varying time-constant $(R C)$, a study was made on the relation between the electric threshold $\left(V_{f}\right)$ of the eye and the radiance $(N)$ of monochromatic rays of differ ent wavelengths used as the adapting light.

(1) In any cases of adapting light of different wavelengths excepting the red light, there were two different types of $V_{f}$ - $\log N$ curve depending upon whether the time-constant was shorter than $25 \mathrm{msec}$. or longer than $75 \mathrm{msec}$. This result was the same as that obtained with the white adapting light.

(2) In the case of time-constant longer than $75 \mathrm{msec}$., the effect of the red adapting light upon $V_{f} \log N$ curve was quite different from that of the other adapting light; the curve showed a form of single nature, whereas in the case of time-constant shorter than $25 \mathrm{msec}$. the same red light had not such specific effect as in the former case.

(3) Taking, as a criterion, the reciprocal of the radiance corresponding to the crest on $V_{f}-\log N$ curve for $0 \mathrm{msec}$ in $R C$ (or the left edge of the horizontal 
part on the curve for $100 \mathrm{msec}$. in $R C$ ), a spectral sensitivity curve was obtained. This curve was proved to be in conformity with scotopic luminosity curve.

(4) It was concluded that the electric threshold, as determined by the exponentially increasing current of time-constant longer than $75 \mathrm{msec}$. was equivalent to the differential threshold of brightness discrimination.

(5) The experiments supported Mita's view that there might be in the retina, at least near the threshold excitation, two sorts of the neural elements which were stimulated separately depending upon the time-constant of the stimulating current. The one for longer time-constant may be the photoreceptor cells, while the other for time-constant the bipolar cells.

\section{ACKNOWLEDGEMENT}

The author wishes to express his deep gratitude to Prof. T. Mita for his constant guidance and valuable advice, and to Dr. A. Akihama for his assistance given to this study.

\section{REFERENCES}

1. Blackwell, H. R. J. Opt. Soc. Amer. 36: 624, 1946.

2. Mita, T., E. Fujimaki And S. YAegashi. Tohoku J. Exp. Med. 65: 45, 1956.

3. MotokawA, K. And K. Iwama. Tohoku J. Exp. Med. 50: 25, 1949.

4. Motokawa, K. Tohoku J. Exp. Med. 54: 385, 1951.

5. Sato, M., T. Suzuki And A. Akihama. Jap. J. Physiol. 7: 29, 1957.

6. Steinhardt, J. J. Gen. Physiol. 20: 185, 1936.

7. Weaver, K. S. J. Opt. Soc. Amer. 39: 278, 1949.

8. YAegashi, S. Tohoku J. Exp. Med. 67: 163, 1958. 